\title{
Two-wave interaction in ideal magnetohydrodynamics
}

\author{
T. V. Zaqarashvili ${ }^{1}$ and B. Roberts ${ }^{2}$ \\ ${ }^{1}$ Georgian National Astrophysical Observatory (Abastumani Astrophysical Observatory), Kazbegi Ave. 2a, Tbilisi 0160, Georgia \\ e-mail: temury@genao.org \\ 2 School of Mathematics and Statistics, University of St. Andrews, St. Andrews, Fife, KY16 9SS, Scotland, UK \\ e-mail: bernie@mcs.st-and.ac.uk
}

Received 3 June 2005 / Accepted 22 February 2006

\section{ABSTRACT}

\begin{abstract}
The weakly nonlinear interaction of sound and linearly polarised Alfvén waves propagating in the same direction along an applied magnetic field is studied. It is found that a sound wave is coupled to the Alfvén wave with double period and wavelength when the sound and Alfvén speeds are equal. The Alfvén wave drives the sound wave through the ponderomotive force, while the sound wave returns energy back to the Alfvén wave through the parametric (swing) influence. As a result, the two waves alternately exchange their energy during propagation. The process of energy exchange is faster for waves with stronger amplitudes. The phenomenon can be of importance in astrophysical plasmas, including the solar atmosphere and solar wind.
\end{abstract}

Key words. magnetohydrodynamics (MHD) - waves

\section{Introduction}

Magnetohydrodynamic (MHD) waves play an important role in various astrophysical situations. The linear description of small amplitude waves is often a good approximation. However, highly dynamical and inhomogeneous astrophysical plasmas may permit the interaction between different MHD wave modes, leading to basically new processes. The energy transformation from one kind of wave into another is of importance in the processes of energy transport and dissipation. MHD wave coupling due to the medium inhomogeneity has been extensively studied in solar-terrestrial connections (Chen \& Hasegawa 1974; Ionson 1978; Rae \& Roberts 1982; Heyvaerts \& Priest 1983; Hollweg 1987; Poedts et al. 1989; Ofman \& Davila 1995). It is also known that in a homogeneous plasma large amplitude circularly polarised Alfvén waves may resonantly drive two other waves, through the three-wave interaction (or decay instability) (Galeev and Oraevsky 1962; Sagdeev \& Galeev 1969). The interaction between various kinds of magnetosonic and Alfvén waves in a uniform plasma has been studied previously (Brodin \& Stenflo 1988). On the other hand, Alfvén waves with varying magnetic field strength (e.g., linearly and elliptically polarised waves) may directly drive density fluctuations through the ponderomotive force (Hollweg 1971; Cohen \& Kulsrud 1974). When the Alfvén speed $v_{\mathrm{A}}$ is different from the sound speed $v_{\mathrm{s}}$, then the frequency and spatial inhomogeneity of generated density fluctuations do not satisfy the dispersion relation of sound waves, and therefore cannot propagate as sound waves, but instead they modify the Alfvén speed. However, when $v_{\mathrm{A}} \approx v_{\mathrm{s}}$, then the density perturbations may propagate as sound waves with a frequency and wave number that is double that of the Alfvén waves. As a result, the nonlinear Schrödinger equation describing the propagation of finite amplitude Alfvén waves along the magnetic field exhibits a discontinuity in this particular case (Spangler \& Sheerin 1982; Medvedev et al. 1997). Medvedev et al. (1997) numerically solved the kinetic nonlinear Schrödinger equation in this case and showed the damping of coherent Alfvén wave trains. However, the back-reaction of generated sound waves (or ion-acoustic waves) on an Alfvén wave is not well studied. We can expect that a nonlinear coupling between Alfvén and sound waves may take place in this particular case, but no clear physical description of the process has so far been given.

On the other hand, the recently proposed new mechanism of MHD swing (wave-wave) interaction (Zaqarashvili 2001; Zaqarashvili \& Roberts 2002; Shergelashvili et al. 2005) can be responsible for the energy transformation from magnetosonic waves into Alfvén waves. The physical basis of this interaction is the parametric influence: the magnetosonic waves cause a periodical variation in the medium parameters, which in turn influences the phase velocity of transversal Alfvén waves and this leads to a resonant energy transformation into certain harmonics. It has been shown that a periodical variation of the medium density, caused by the propagation of sound waves along an applied magnetic field in a high $\beta$ plasma $\left(\beta=8 \pi p / B^{2}\right.$, where $p$ is the plasma pressure and $B$ is the magnetic field strength), results in Alfvén waves governed by the Mathieu equation (Zaqarashvili 2001). Consequently, harmonics with half the frequency of sound waves grow exponentially in time. The phenomenon has been developed for the case of fast magnetosonic waves propagating across a magnetic field and Alfvén waves propagating along the field (Zaqarashvili \& Roberts 2002; Shergelashvili et al. 2005). The process of energy exchange between these different kinds of wave motion is called swing wave-wave interaction (Zaqarashvili \& Roberts 2002). This terminology arises by analogy with a spring pendulum. It can be shown that the transversal (pendulum) and longitudinal (spring) oscillations are coupled when the eigenfrequency of transversal oscillations is half the frequency of the spring oscillations.

Taking into account the swing wave-wave interaction, one may suggest that sound waves resonantly drive the harmonics of Alfvén waves with half frequency and wave number when $v_{\mathrm{A}} \approx v_{\mathrm{s}}$. Consequently, the waves may alternately exchange energy as they propagate along an applied magnetic field. A sound 
wave entering a region where $v_{\mathrm{A}} \approx v_{\mathrm{s}}$ may resonantly drive an Alfvén wave, and vice versa. Also, the plasma $\beta$ approaches to unity in parts of the solar wind plasma, and in the lower solar atmosphere there is a region where the Alfvén and sound speeds are approximately equal (Gary 2001; see Fig. 3 of that paper). Moreover, the recent observations by Muglach et al. (2005) suggest a possible transformation of compressible wave energy into incompressible waves in this particular region. Therefore the process of Alfvén and sound wave coupling at $v_{\mathrm{A}} \approx v_{\mathrm{s}}$ can be of general importance in the solar atmosphere, solar wind and other astrophysical situations.

In the next section we consider analytically the weakly nonlinear MHD equations, giving a short description of some astrophysical situations where the process can be of importance.

\section{Wave interactions}

Consider fluid motions $\boldsymbol{u}$ in a magnetised medium (with zero viscosity and infinite electrical conductivity), as described by the ideal MHD equations:

$$
\begin{aligned}
& \frac{\partial \boldsymbol{B}}{\partial t}+(\boldsymbol{u} \cdot \nabla) \boldsymbol{B}=(\boldsymbol{B} \cdot \nabla) \boldsymbol{u}-\boldsymbol{B} \nabla \cdot \boldsymbol{u}, \quad \nabla \cdot \boldsymbol{B}=0, \\
& \rho \frac{\partial \mathbf{u}}{\partial t}+\rho(\boldsymbol{u} \cdot \nabla) \boldsymbol{u}=-\nabla\left[p+\frac{B^{2}}{8 \pi}\right]+\frac{(\boldsymbol{B} \cdot \nabla) \boldsymbol{B}}{4 \pi}, \\
& \frac{\partial \rho}{\partial t}+(\boldsymbol{u} \cdot \nabla) \rho+\rho \nabla \cdot \boldsymbol{u}=0, \\
& \frac{\partial p}{\partial t}+(\boldsymbol{u} \cdot \nabla) p+\gamma p \nabla \cdot \boldsymbol{u}=0,
\end{aligned}
$$

where $p$ is the pressure, $\rho$ is the density, $\boldsymbol{B}$ is the magnetic field and $\gamma$ is the ratio of specific heats. We neglect gravity, though it may be of importance in some astrophysical situations.

Consider the case of a homogeneous medium with an uniform magnetic field $\boldsymbol{B}=\left(B_{0}, 0,0\right)$ directed along the $x$ axis of a Cartesian coordinate system. We consider wave propagation along the $x$ axis (thus along the magnetic field) and wave polarisation in $x y$ plane. In this case, two kinds of wave may propagate strictly along the applied magnetic field: sound and linearly polarised Alfvén waves. In the linear limit these waves are strictly different; the Alfvén waves are purely transversal with the velocity component along the $y$ axis (magnetic tension provides the restoring force), while the sound waves are purely longitudinal with the velocity component along the $x$ axis (pressure gradients provide the restoring force). In this case the energy exchange between waves results from nonlinear interactions. As velocity and magnetic field components of linearly polarised Alfvén waves lie in $x y$ plane, then we may consider the two dimensional MHD equations.

In principle, the transverse inhomogeneity of the magnetic field along the $z$-axis can also be taken into account. In this case each magnetic $x y$ surface will behave independently (when the waves propagate strictly along the applied field and the dissipation is neglected). Therefore we may again consider two dimensional equations for each magnetic $x y$ surface separately. However, here we consider the homogeneous medium, keeping in mind that similar processes can also occur in inhomogeneous (transverse to the magnetic field) plasmas.

As we consider wave propagation along the $x$ axis, then the $x$ and $y$ components of Eqs. (1)-(4) take the form:

$$
\frac{\partial b_{y}}{\partial t}+u_{x} \frac{\partial b_{y}}{\partial x}+b_{y} \frac{\partial u_{x}}{\partial x}-B_{0} \frac{\partial u_{y}}{\partial x}=0,
$$

$$
\begin{aligned}
& \rho \frac{\partial u_{y}}{\partial t}+\rho u_{x} \frac{\partial u_{y}}{\partial x}-\frac{B_{0}}{4 \pi} \frac{\partial b_{y}}{\partial x}=0 \\
& \frac{\partial \rho}{\partial t}+\rho \frac{\partial u_{x}}{\partial x}+u_{x} \frac{\partial \rho}{\partial x}=0 \\
& \rho \frac{\partial u_{x}}{\partial t}+\rho u_{x} \frac{\partial u_{x}}{\partial x}+\frac{\partial p}{\partial x}+\frac{\partial}{\partial x} \frac{b_{y}^{2}}{8 \pi}=0 \\
& \frac{\partial p}{\partial t}+\gamma p \frac{\partial u_{x}}{\partial x}+u_{x} \frac{\partial p}{\partial x}=0
\end{aligned}
$$

where $p$ and $\rho$ denote the total pressure and density, $u_{y}$ and $u_{x}$ are the velocity perturbations (of the Alfvén and sound waves, respectively), and $b_{y}$ is the perturbation in the magnetic field. These equations describe the fully nonlinear behaviour of sound and linearly polarised Alfvén waves propagating along an applied magnetic field.

For a mechanical analogy of the wave coupling process, we recall the pendulum with stiffness spring (Zaqarashvili \& Roberts 2002). There are two different oscillations of such a pendulum: transversal oscillations are due to gravity, and longitudinal oscillations are due to the stiffness force of the elastic spring. Transversal oscillations influence the longitudinal ones, due to the varying gravitational field component acting along the pendulum axis, while longitudinal oscillations influence transversal ones parametrically through the variation of the pendulum length. When the eigenfrequency of the transversal oscillation is half the frequency of the spring oscillation, then a resonant coupling occurs. Initial transversal oscillations directly drive longitudinal oscillations and they return the energy back to transversal oscillations through the parametric influence. The energy exchange between the oscillations occurs alternately, without dissipation.

A similar process occurs in the case of Alfvén and sound waves when they propagate with the same phase speeds along an applied magnetic field. If initially there is an Alfvén wave, then it drives sound waves with twice the frequency and wave number of the Alfvén wave through the ponderomotive force (Hollweg 1971). A more interesting and new process occurs when initially there is a sound wave: then small transversal perturbations can be amplified through the parametric action of the sound wave (Zaqarashvili 2001). The Alfvén wave results from the Lorentz force acting against the fluid inertia. The periodical compression (expansion) of the medium due to the sound wave reinforces the fluid inertia in certain phases and this leads to the amplification of an Alfvén wave with half the frequency and wave number of the sound wave (see Fig. 1). The parametric influence of sound waves is expressed through the nonlinear terms in Eqs. (5) and (6).

We express the total plasma density and pressure as the sum of the unperturbed and perturbed parts, $\rho_{0}+\rho_{1}$ and $p_{0}+p_{1}$, respectively, and consider the weakly nonlinear process when the perturbations are much smaller than the unperturbed values. Then the variation of wave amplitudes, due to nonlinear interactions, will be a slow process. The perturbations can be represented as the product of a slowly varying amplitude $C_{j}(t)$ (with complex conjugate $\left.C_{j}^{*}(t)\right)$ and a rapidly oscillating term:

$$
\begin{aligned}
& \rho_{1}=C_{1}(t) \mathrm{e}^{\mathrm{i} \phi_{2}(t, x)}+C_{1}^{*}(t) \mathrm{e}^{-\mathrm{i} \phi_{2}(t, x)}, \\
& u_{x}=C_{2}(t) \mathrm{e}^{i \phi_{2}(t, x)}+C_{2}^{*}(t) \mathrm{e}^{-\mathrm{i} \phi_{2}(t, x)}, \\
& b_{y}=C_{3}(t) \mathrm{e}^{\mathrm{i} \phi_{1}(t, x)}+C_{3}^{*}(t) \mathrm{e}^{-\mathrm{i} \phi_{1}(t, x)}, \\
& u_{y}=C_{4}(t) \mathrm{e}^{\mathrm{i} \phi_{1}(t, x)}+C_{4}^{*}(t) \mathrm{e}^{-\mathrm{i} \phi_{1}(t, x)}, \\
& p_{1}=C_{5}(t) \mathrm{e}^{\mathrm{i} \phi_{2}(t, x)}+C_{5}^{*}(t) \mathrm{e}^{-\mathrm{i} \phi_{2}(t, x)}
\end{aligned}
$$




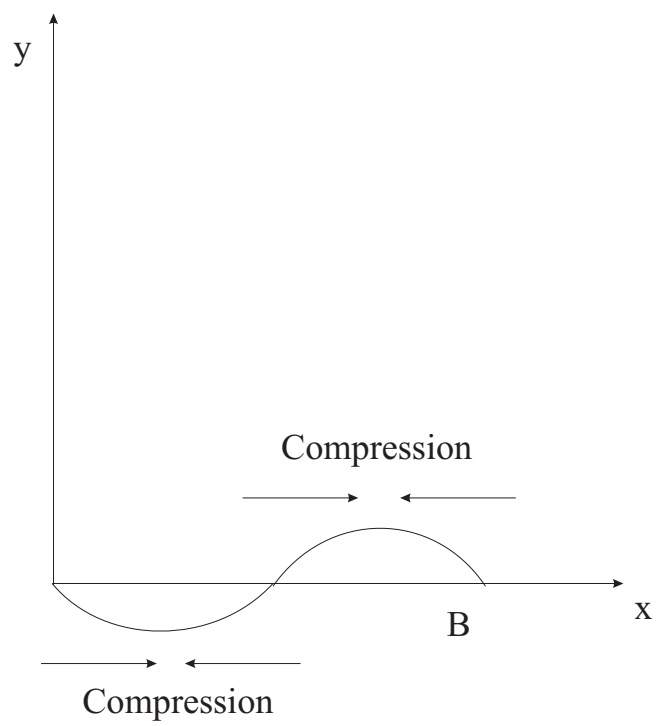

Fig. 1. The influence of a sound wave on an Alfvén wave with double period and wavelength. The periodical compression (expansion) of the medium due to the sound wave may amplify the Alfvén wave. The double period of the Alfvén wave arises due to the displacement of plasma elements on both sides of the magnetic field.

Here

$\phi_{1}=\omega_{\mathrm{A}} t-k_{\mathrm{A}} x, \quad \phi_{2}=\omega_{\mathrm{s}} t-k_{\mathrm{s}} x$,

where $\omega_{\mathrm{A}}, k_{\mathrm{A}}, \omega_{\mathrm{s}}, k_{\mathrm{s}}$ are the frequencies and wave numbers of Alfvén and sound waves, respectively. The frequencies and wave numbers satisfy the dispersion relations for sound and Alfvén waves: $\omega_{\mathrm{s}} / k_{\mathrm{s}}=v_{\mathrm{s}}$ and $\omega_{\mathrm{A}} / k_{\mathrm{A}}=v_{\mathrm{A}}$, where $v_{\mathrm{s}}=\sqrt{\gamma p_{0} / \rho_{0}}$ and $v_{\mathrm{A}}=B_{0} / \sqrt{4 \pi \rho_{0}}$ are the sound and Alfvén speeds respectively.

In general, the amplitudes $C_{j}$ can be slowly varying functions of the $x$ coordinate too. However, for simplicity we neglect the $x$ dependence and look to their temporal evolution (see Sagdeev \& Galeev 1969 for a similar approximation).

Substitution of expressions (10) into Eqs. (5)-(9), and averaging over rapid oscillations in $x$ and $t$ (as both phases $\phi_{1}$ and $\phi_{2}$ include these variables), leads to the cancelling of all exponential terms (after averaging, harmonic functions become zero), so that only the first and third order (in $C_{j}$ ) terms remain. In the first approximation (neglecting all third order terms), the sound and Alfvén waves are decoupled and the amplitudes $C_{j}$ are constant. Third order terms with $C_{j}$ (which are due to the advective terms in the momentum equation) are significant only in the case of very large amplitudes and presumably lead to the steepening of wave fronts and consequently to the formation of shock waves. However, if frequencies and wave numbers satisfy the conditions

$\omega_{\mathrm{s}}=2 \omega_{\mathrm{A}}, \quad k_{\mathrm{s}}=2 k_{\mathrm{A}}$,

or $\omega_{\mathrm{s}}=-2 \omega_{\mathrm{A}}$ and $k_{\mathrm{s}}=-2 k_{\mathrm{A}}$, then after averaging the second order terms (with $C_{j}$ ) remain and wave amplitudes become time dependent. These conditions are fulfilled when

$v_{\mathrm{A}}=v_{\mathrm{S}}$.

Therefore the sound and Alfvén waves are resonantly coupled and so may exchange energies when they propagate with the same speed along the magnetic field. If wave phase speeds vary across the magnetic field, then the wave coupling may take place in a particular resonant layer where condition (13) is satisfied.

With conditions (12) and (13), the averaging of Eqs. (5)-(9) over rapid oscillations in $x$ and $t$ leads to equations which govern the temporal behaviour of complex amplitudes (with the third order terms in $C_{j}$ neglected):

$$
\begin{gathered}
\frac{\mathrm{d} C_{3}}{\mathrm{~d} t}+\mathrm{i} \omega_{\mathrm{A}} C_{3}+\mathrm{i} k_{\mathrm{A}} B_{0} C_{4}-\mathrm{i} k_{\mathrm{A}} C_{2} C_{3}^{*}=0, \\
\frac{\mathrm{d} C_{4}}{\mathrm{~d} t}+\mathrm{i} \omega_{\mathrm{A}} C_{4}+\mathrm{i} \frac{B_{0}}{4 \pi \rho_{0}} k_{\mathrm{A}} C_{3}+\frac{C_{1}}{\rho_{0}} \frac{\mathrm{d} C_{4}^{*}}{\mathrm{~d} t} \\
-\mathrm{i} \omega_{\mathrm{A}} \frac{C_{1}}{\rho_{0}} C_{4}^{*}+\mathrm{i} k_{\mathrm{A}} C_{2} C_{4}^{*}=0,
\end{gathered}
$$

$\frac{\mathrm{d} C_{1}}{\mathrm{~d} t}+2 \mathrm{i} \omega_{\mathrm{A}} C_{1}-2 \mathrm{i} k_{\mathrm{A}} \rho_{0} C_{2}=0$,

$\frac{\mathrm{d} C_{2}}{\mathrm{~d} t}+2 \mathrm{i} \omega_{\mathrm{A}} C_{2}-2 \mathrm{i} k_{\mathrm{A}} v_{\mathrm{s}}^{2} \frac{C_{1}}{\rho_{0}}-\frac{2 \mathrm{i} k_{\mathrm{A}} C_{3}^{2}}{8 \pi \rho_{0}}=0$.

These equations describe the process of energy exchange between the sound and Alfvén waves. The influence of the ponderomotive force is expressed by the last term in Eq. (17) and the parametric influence of the sound wave is expressed through the last term in Eq. (14) and the last three terms in Eq. (15).

From Eqs. (14)-(17) we obtain two second order differential equations by eliminating $C_{2}$ and $C_{4}$ in favour of $C_{1}$ and $C_{3}$ (third order terms in $C_{j}$ being neglected):

$$
\begin{aligned}
\frac{\mathrm{d}^{2} C_{3}}{\mathrm{~d} t^{2}}+ & 2 \mathrm{i} \omega_{\mathrm{A}} \frac{\mathrm{d} C_{3}}{\mathrm{~d} t}-\frac{1}{2 \rho_{0}} C_{3}^{*} \frac{\mathrm{d}^{2} C_{1}}{\mathrm{~d} t^{2}}-\frac{1}{\rho_{0}} \frac{\mathrm{d} C_{1}}{\mathrm{~d} t} \frac{\mathrm{d} C_{3}^{*}}{\mathrm{~d} t} \\
& -\frac{\mathrm{i} \omega_{\mathrm{A}}}{\rho_{0}} C_{3}^{*} \frac{\mathrm{d} C_{1}}{\mathrm{~d} t}+\frac{\omega_{\mathrm{A}}^{2}}{\rho_{0}} C_{1} C_{3}^{*}-\frac{1}{\rho_{0}} C_{1} \frac{\mathrm{d}^{2} C_{3}^{*}}{\mathrm{~d} t^{2}}=0
\end{aligned}
$$

and

$\frac{\mathrm{d}^{2} C_{1}}{\mathrm{~d} t^{2}}+4 \mathrm{i} \omega_{\mathrm{A}} \frac{\mathrm{d} C_{1}}{\mathrm{~d} t}+\frac{k_{\mathrm{A}}^{2}}{2 \pi} C_{3}^{2}=0$.

Analytical solution of these equations is still complicated. However, if the amplitude of one wave is considered to be stronger than the other, then a significant simplification can be achieved. In this case the back reaction can be ignored (at least at the initial stage) and the amplitude of the initial wave becomes constant. This allows us to study the influence of the pumping wave on the evolution of the other wave.

We begin by studying the general behaviour of Eqs. (18) and (19), using a phase-plane analysis. As $C_{1}$ and $C_{3}$ are slowly varying functions in time, we may neglect the second derivatives and third order terms in $C_{3}$. Then

$2 \mathrm{i} \omega_{\mathrm{A}} \frac{\mathrm{d} C_{3}}{\mathrm{~d} t}+\frac{\omega_{\mathrm{A}}^{2}}{\rho_{0}} C_{1} C_{3}^{*}=0$

$4 \mathrm{i} \omega_{\mathrm{A}} \frac{\mathrm{d} C_{1}}{\mathrm{~d} t}+\frac{k_{\mathrm{A}}^{2}}{2 \pi} C_{3}^{2}=0$.

Writing $C_{1}=C_{10}+\mathrm{i} C_{11}$ and $C_{3}=C_{30}+\mathrm{i} C_{31}$, we note that the equations simplify if we choose the initial phase of the Alfvén waves so that $C_{30}=C_{31}$. Then, from Eq. (21), we obtain $C_{10}=0$. Writing $A=C_{10} / \rho_{0}$ and $B=C_{30} / B_{0}$, we have

$\frac{\mathrm{d} A}{\mathrm{~d} t}=-\omega_{\mathrm{A}} B^{2}(t)$

$\frac{\mathrm{d} B}{\mathrm{~d} t}=\frac{1}{2} \omega_{\mathrm{A}} A(t) B(t)$. 




Fig. 2. Phase plane diagram for $A=C_{10} / \rho_{0}$ (sound mode) and $B=$ $C_{30} / B_{0}$ (Alfvén mode) as described by Eqs. (22) and (23). Equilibrium points of the system are the origin and the entire $A$ axis. Each phase path corresponds to a particular possible development of the system, indicated by an arrow. It is seen that even a very small displacement of the system from the equilibrium points (i.e. from the $A$ axis) results in further developments. Physically, it means that a small transversal perturbation causes an energy transfer from the sound wave into the Alfvén wave. When $B$ reaches a maximum, the entire energy of the system is stored in Alfvén waves. Then the energy is once more transferred into the sound wave. Note that if we draw the same phase diagram for $\sqrt{\left(C_{30}^{2}+C_{31}^{2}\right) / B_{0}^{2}}=\sqrt{2} B$ (which is the real amplitude of Alfvén wave), then the ellipse transforms into a circle.

These equations describe a so called autonomous system as the time variable does not appear independently of A and B in the right-handside of the equations. The general behaviour of such a system is revealed by phase-plane analysis (see Jordan \& Smith 1987 , pp. 36-38). The solutions $A(t), B(t)$ may be represented on a plane with Cartesian axes $A, B$. Then as $t$ increases the point $(A(t), B(t))$ traces out a directed curve in the plane; this is a phase path. From Eqs. (22) and (23) we get:

$\frac{\mathrm{d} B}{\mathrm{~d} A}=-\frac{1}{2} \frac{A}{B}$,

which integrates to

$A^{2}+2 B^{2}=$ const.

The phase diagram of the system is plotted in Fig. 2. Note that the equilibrium points of this system (where the right-handside terms in Eqs. (22) and (23) become zero) are the origin together with the entire $A$ axis (shown by a thick line). Each phase path corresponds to a particular possible development of the system, indicated by an arrow in Fig. 2, showing how the state of the system changes as time increases. For example, if the system is initially in equilibrium (i.e. only $A$ exists and $B$ is zero), then even a very small displacement in $B$ leads to an evolution in the system: $B$ increases and $A$ decreases. When $B$ reaches to its maximum, $A$ becomes zero. Then the system continues development and reaches again to the $A$ axis.

The potential energy density of a sound wave is $E_{\mathrm{s}}=$ $C_{10}^{2} / \rho_{0}^{2}=A^{2}$ and the energy density of an Alfvén wave is


Fig. 3. The long term evolution of wave energy $E_{\mathrm{s}}$ in the sound mode and the wave energy $E_{\mathrm{A}}$ in the Alfvén mode. Initially the energy is stored in the Alfvén wave $\left(E_{\mathrm{s}}=0\right)$. Subsequently it transforms into the energy of a sound wave. An alternating energy exchange between the waves then ensues. The process is averaged by rapid oscillations.

$E_{\mathrm{A}}=\left(C_{30}^{2}+C_{30}^{2}\right) / B_{0}^{2}=2 B^{2}$. Thus the phase diagram shows that the waves continually exchange their energies: if initially there is a sound wave, then the small transverse displacement causes an energy transformation into an Alfvén wave, and vice versa. Equation (25) shows that the sum of wave energies remains constant, as suggested on physical ground (in the absence of dissipation).

We now turn to the long term evolution of sound $E_{\mathrm{s}}$ and Alfvén $E_{\mathrm{A}}$ wave energies, derived from a numerical solution of the differential equation system (14)-(17). The results are plotted in Fig. 3. Only an Alfvén wave exists initially. Subsequently, its energy transforms into the sound wave before later returning back to the Alfvén wave, much as predicted analytically. In the absence of dissipation, the waves exchange their energies alternately.

The numerical solution of Eqs. (14)-(17) shows that the time scale of energy exchange depends on the wave amplitudes; it is shorter for waves with stronger amplitudes. The dependence of $\delta / \omega_{\mathrm{A}}$ (the ratio of the energy transfer frequency $\delta$ to the wave frequency $\omega_{\mathrm{A}}$ ) on the wave amplitude, derived by numerical integration of system (14)-(17), is displayed in Fig. 4.

Thus, as demonstrated both analytically and numerically, there is a continuous energy exchange between sound and Alfvén waves. The energy transfer occurs in both ways: an initial sound wave transfers its energy to an Alfvén wave, and vice versa.

Now let consider the sound wave to be the pump, i.e. the amplitude of the sound wave is much stronger than the initial amplitude of the Alfvén wave. Then the back reaction can be ignored with $C_{1}$ taken to be constant. Under these circumstances Eq. (18) takes the form:

$\frac{\mathrm{d}^{2} C_{3}}{\mathrm{~d} t^{2}}+2 \mathrm{i} \omega_{\mathrm{A}} \frac{\mathrm{d} C_{3}}{\mathrm{~d} t}+\frac{\omega_{\mathrm{A}}^{2}}{\rho_{0}} C_{1} C_{3}^{*}-\frac{1}{\rho_{0}} C_{1} \frac{\mathrm{d}^{2} C_{3}^{*}}{\mathrm{~d} t^{2}}=0$

We search for solutions of the form

$C_{1}=C_{10}+\mathrm{i} C_{11}$,

$C_{3}=\left(C_{30}+\mathrm{i} C_{31}\right) \mathrm{e}^{\delta t}$, 


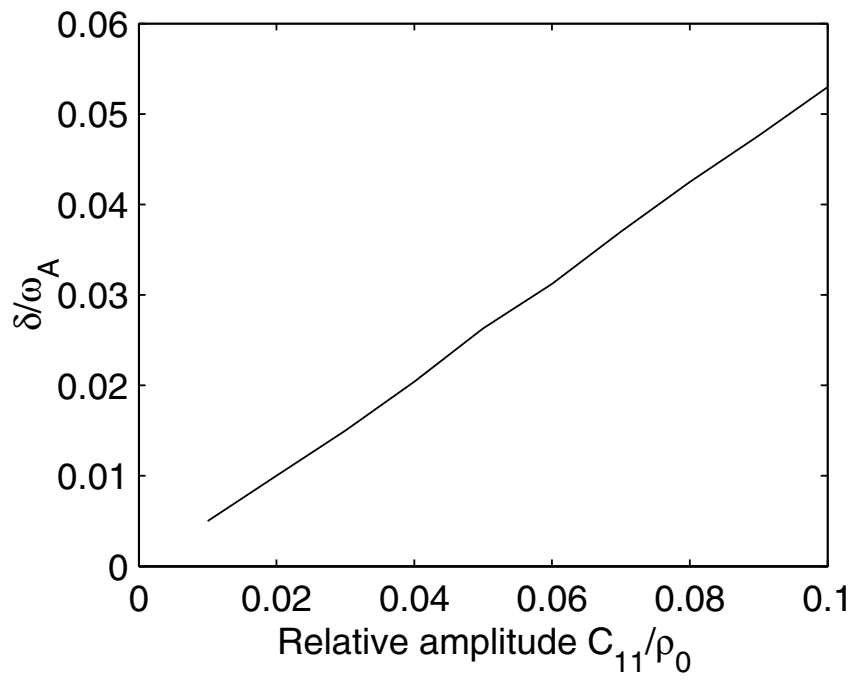

Fig. 4. The dependence of $\delta / \omega_{\mathrm{A}}$, the ratio of the modulation frequency $\delta$ and wave frequency $\omega_{\mathrm{A}}$, on the wave amplitude derived by numerical calculations of the system (14)-(17). The time scale of energy exchange is shorter for strong amplitude waves.

where $C_{10}, C_{11}, C_{30}$ and $C_{31}$ are constants.

Substituting expressions (27) and (28) into Eq. (26), and for simplicity considering $C_{11}=0$, we obtain a fourth order equation for $\delta$ :

$\left(1-\frac{C_{10}^{2}}{\rho_{0}^{2}}\right) \delta^{4}+2 \omega_{\mathrm{A}}^{2}\left(2+\frac{C_{10}^{2}}{\rho_{0}^{2}}\right) \delta^{2}-\omega_{\mathrm{A}}^{4} \frac{C_{10}^{2}}{\rho_{0}^{2}}=0$.

Then $C_{3}$ has an exponentially growing solution with the growth rate

$\delta \approx \frac{1}{2} \frac{C_{10}}{\rho_{0}} \omega_{\mathrm{A}}$.

Thus the pump sound wave causes an exponential amplification of the Alfvén wave amplitude. Energy transfers from the initial pump sound wave to the Alfvén wave. The reverse process, i.e. energy transformation from a pump Alfvén wave into sound waves, was first studied by Hollweg (1971).

Numerical solution of the system (14)-(17) when $C_{1}$ and $C_{2}$ are constants also shows an exponential amplification of $C_{3}$ and $C_{4}$, as suggested by the analytical solution (see Eqs. (28) and (30)). The results of a numerical solution, together with the analytical expression (30), are plotted in Fig. 5. It is seen that the analytical and numerical solutions are indistinguishable (given the same initial conditions).

Here we have solved numerically only the system (14)-(17), obtained after averaging by rapid oscillation. In future it would be interesting to investigate the complete numerical simulation of Eqs. (5)-(9).

\section{Discussion}

We have studied the weakly nonlinear interaction between sound and linearly polarised Alfvén waves propagating along an applied magnetic field. We have shown that after averaging of the nonlinear MHD equations for rapid oscillations the wave amplitudes become time dependent if the frequencies and wave numbers satisfy the resonant conditions $\omega_{\mathrm{s}}= \pm 2 \omega_{\mathrm{A}}$ and $k_{\mathrm{s}}= \pm 2 k_{\mathrm{A}}$, where $\omega_{\mathrm{A}}, k_{\mathrm{A}}$ and $\omega_{\mathrm{s}}, k_{\mathrm{s}}$ are the frequencies and wave numbers of Alfvén and sound waves, respectively. These conditions are fulfilled when the phase speeds of Alfvén and sound



Fig. 5. The plots of analytical (solid line) and numerical (dashed line) expressions of Alfvén wave amplitude are presented here when the sound wave is considered as the pumping wave. The relative amplitude of sound wave $C_{10} / \rho_{0}$ is 0.1 , while initial relative amplitude of the Alfvén wave $C_{30} / B_{0}$ is 0.01 . The time is normalised by the period of Alfvén wave, $t_{\mathrm{A}}$. The exponential growth of Alfvén wave amplitude is identical in both cases.

waves are equal, so that $v_{\mathrm{A}} \approx v_{\mathrm{s}}$. Then the waves propagating in the same direction along the magnetic field alternately exchange energy (see Figs. 2 and 3). The timescale of energy exchange depends on the wave amplitude (see Fig. 4); strong amplitude waves cause a faster process of energy exchange. Thus the long standing uncertainty in the $\beta=1$ case in nonlinear MHD may be resolved by resonant nonlinear coupling between Alfvén and sound waves. However, generally astrophysical plasmas are highly inhomogeneous, requiring a treatment of this case. In principle, a calculation along the lines described here for a homogeneous plasma can also be done in the case of a transversally inhomogeneous medium. Then the coupling may take place when the waves propagate in a resonant layer where $v_{\mathrm{A}} \approx v_{\mathrm{s}}$.

Note that here there are only two coupled waves: sound and linearly polarised Alfvén waves propagating in the same direction along the applied magnetic field. Therefore this process is basically different from the well known three-wave interaction (Galeev \& Oraevsky 1962; Sagdeev \& Galeev 1969). We call it swing interaction or two-wave interaction. Here the Alfvén wave drives the sound wave, due to the nonlinear magnetic pressure, while the sound wave returns the energy back to the Alfvén wave through parametric action (Zaqarashvili 2001; Zaqarashvili \& Roberts 2002). The physics of the parametric (swing) influence is simple: the sound wave causes a periodical variation of the medium's plasma density, i.e. the fluid inertia, and consequently works against the tension force (the restoring force for an Alfvén wave). The harmonics of an Alfvén wave with half the frequency of a sound wave grow in time, as in the case of a pendulum with periodically varying length. The half frequency arises due to the displacement of plasma elements on both sides of the magnetic field (see Fig. 1).

Here we have shown that the wave coupling occurs in the region where the waves propagate with the same speed, $v_{\mathrm{A}}=v_{\mathrm{s}}$, along the magnetic field. However, it is well known that any resonant process has a resonant interval of frequencies, the width of which depends on the wave amplitudes. Therefore the sound and Alfvén waves with relatively stronger amplitudes may be coupled even in the case when $v_{\mathrm{A}} \neq v_{\mathrm{s}}$. Thus the width of resonant 
layer will be wider for stronger amplitude waves. Also, a similar phenomenon may arise between Alfvén and obliquely propagating fast magnetosonic waves. But these processes require further study.

It must be mentioned that numerical simulations of wave propagation in a two-dimensional stratified magneto-atmosphere show the coupling between MHD waves in the region where the sound and Alfvén speeds are comparable in magnitude (Rosenthal et al. 2002; Bogdan et al. 2003). This very interesting result is different from our consideration as the coupling in those papers occurs between fast and slow magnetoacoustic-gravity waves. It would be interesting to carry out numerical simulations in order to test the coupling between sound and Alfvén waves suggested in our paper.

It may be noted that the method of slowly varying amplitudes used here does not include the process of wave steepening due to the generation of higher harmonics. Consequently, it describes the energy transformation process only in the early stages of wave coupling. The generation of higher harmonics may modify the situation. However, electron dispersion effects in two fluid MHD may act against steepening and then the process of coupling will remain unchanged.

Recent modelling of the plasma $\beta$ in the solar atmosphere (Gary 2001) shows that $v_{\mathrm{A}} \sim v_{\mathrm{s}}$, i.e. $\beta \sim 1$, may takes place not only in the lower chromosphere, but also at relatively low coronal heights. Thus the suggested wave coupling can be of importance in that part of the solar atmosphere, where $v_{\mathrm{A}} \sim v_{\mathrm{s}}$, and in the solar wind.

It is generally considered that solar 5-minute acoustic oscillations ( $p$-modes) cannot penetrate into the corona due to the sharp temperature gradient in the transition region. However, 5-min intensity oscillations are intensively observed in the corona by the space satellites SOHO (Solar and Heliospheric Observatory) and TRACE (Transition Region and Coronal Explorer) (De Moortel et al. 2002), and recently De Pontieu et al. (2004, 2005) have discussed how photospheric oscillations can be channelled into the corona through inclined magnetic fields. Our proposed mechanism of wave coupling may also resolve this problem. The acoustic oscillations may transform their energy into Alfvén waves, or possibly into surface kink waves in thin photospheric magnetic tubes, this process acting in the region of the solar atmosphere where $v_{\mathrm{A}} \approx v_{\mathrm{s}}$. Generated transversal waves may then propagate through the transition region into the corona, where they can deposit their energy back into density perturbations. The process can be thus be of importance in coronal heating. It is interesting to note that MHD oscillations with the properties of Alfvén waves have been observed in the photosphere and lower chromosphere by Ulrich (1996). He found that the power spectrum of the magnetic variations includes substantial power at frequencies lower than the $5 \mathrm{~min}$ oscillation (see Fig. 3 of that paper). This may be caused by our energy conversion mechanism. It is also interesting to note the recent observations by Muglach et al. (2005), which suggest a possible transformation of compressible wave energy into incompressible waves in the $\beta \approx 1$ region of the solar atmosphere.
Finally, we note that the two-wave interaction process can also be of importance as an explanation of the observed rotational discontinuities and pressure-balanced structures found in the solar wind (Vasquez \& Hollweg 1996, 1999). Swing coupling between Alfvén and obliquely propagating fast magnetosonic waves may lead to the formation of these structures, and this in turn can be of importance in the problem of solar wind acceleration.

\section{Conclusions}

We have described the nonlinear coupling between sound and linearly polarised Alfvén waves propagating with the same speed along an applied magnetic field. The sound wave is coupled to the Alfvén wave with a period and wavelength that is double that of the Alfvén wave. Analytical and numerical solutions show that the waves alternately exchange their energies during propagation. The phenomenon also can be of importance (after appropriate modifications) in the solar atmosphere and solar wind as well as in various other astrophysical and laboratory situations.

Acknowledgements. We thank Professors J. V. Hollweg (UNH) and M. S. Ruderman (Sheffield) for helpful discussions. The work of T. Z. was partially supported by the NATO Reintegration Grant FEL.RIG 980755 and MCyT grant AYA2003-00123.

\section{References}

Bogdan, T. J., Hansteen, M., Carlsson, V., et al. 2003, ApJ, 599, 626 Brodin, G., \& Stenflo, L. 1988, J. Plasma Phys., 39, 277

Chen, L., \& Hasegawa, A. 1974, Phys. Fluids, 17, 1399

Cohen, R. H., \& Kulsrud, R. M. 1974, Phys. Fluids, 17, 2215

De Moortel, I., Ireland, J., Hood, A. W., \& Walsh, R. W. 2002, A\&A, 387, L13

De Pontieu, B., Erdelyi, R., \& James, S. P. 2004, Nature, 430, 536

De Pontieu, B., Erdelyi, R., \& De Moortel, I. 2005, ApJ, 624, L61

Galeev, A. A., \& Oraevsky, V. N. 1962, Sov. Phys. Dokl., 7, 988

Gary, G. A. 2001, Sol. Phys., 203, 71

Heyvaerts, J., \& Priest, E. R. 1983, A\&A, 117, 220

Hollweg, J. V. 1971, J. Geophys. Res., 76, 5515

Hollweg, J. V. 1987, ApJ, 317, 514

Ionson, J. A. 1978, ApJ, 226, 650

Jordan, D. W., \& Smith, P. 1987, Nonlinear Ordinary Differential Equations (Oxford: Clarendon Press)

Medvedev, M. V., Diamond, P. H., Shevchenko, V. I., \& Galinsky, V. L. 1997, Phys. Rev. Lett., 78, 4934

Muglach, K., Hofmann, A., \& Staude, J. 2005, A\&A, 437, 1055

Ofman, L., \& Davila, J. M. 1995, J. Geophys. Res., 100, 23427

Poedts, S., Goossens, M., \& Kerner, W. 1989, Sol. Phys., 123, 83

Rae, I. C., \& Roberts, B. 1982, MNRAS, 201, 1171

Rosenthal, C. S., Bogdan, T. J., Carlsson, M., et al. 2002, ApJ, 564, 508

Sagdeev, R. Z., \& Galeev, A. A. 1969, Nonlinear Plasma Theory (New York: W.A. Benjamin)

Shergelashvili, B. M., Zaqarashvili, T. V., Poedts, S., \& Roberts, B. 2005, A\&A, 429, 767

Spangler, S. R., \& Sheerin, J. P. 1982, J. Plasma Phys., 27, 193

Ulrich, R. K. 1996, ApJ, 465, 436

Vasquez, B. J., \& Hollweg, J. V. 1996, J. Geophys. Res., 101, 13527

Vasquez, B. J., \& Hollweg, J. V. 1999, J. Geophys. Res., 104, 4681

Zaqarashvili, T. V. 2001, ApJ, 552, L81

Zaqarashvili, T. V., \& B. Roberts, 2002, Phys. Rev. E, 66, 026401 